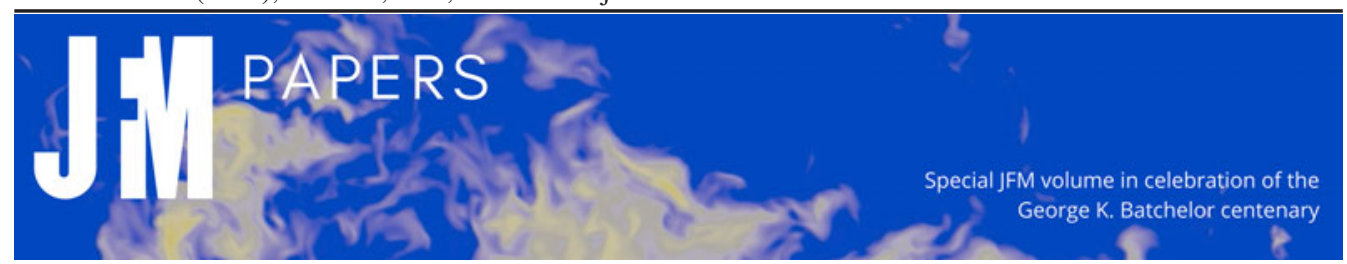

\title{
Numerical and asymptotic analysis of the three-dimensional electrohydrodynamic interactions of drop pairs
}

\author{
Chiara Sorgentone ${ }^{1}$, Jeremy I. Kach ${ }^{2}$, Aditya S. Khair ${ }^{2}$, Lynn M. Walker ${ }^{2}$ and \\ Petia M. Vlahovska ${ }^{3, \dagger}$ \\ ${ }^{1}$ KTH Mathematics, Linné Flow Centre, 10044 Stockholm, Sweden \\ ${ }^{2}$ Department of Chemical Engineering, Carnegie Mellon University, Pittsburgh PA 15213, USA \\ ${ }^{3}$ Engineering Sciences and Applied Mathematics, Northwestern University, Evanston, IL 60208, USA
}

(Received 11 April 2020; revised 8 October 2020; accepted 5 November 2020)

We study the pairwise interactions of drops in an applied uniform DC electric field within the framework of the leaky dielectric model. We develop three-dimensional numerical simulations using the boundary integral method and an analytical theory assuming small drop deformations. We apply the simulations and the theory to explore the electrohydrodynamic interactions between two identical drops with arbitrary orientation of their line of centres relative to the applied field direction. Our results show a complex dynamics depending on the conductivities and permittivities of the drops and suspending fluids, and the initial drop pair alignment with the applied electric field.

Key words: electrohydrodynamic effects, boundary integral methods

\section{Introduction}

The interaction of fluids and electric fields is at the heart of natural phenomena such as the disintegration of raindrops in thunderstorms and many practical applications such as electrosprays (Ganan-Calvo et al. 2018), microfluidics (Stone, Stroock \& Ajdari 2005) and crude oil demulsification (Eow \& Ghadiri 2002). Many of these processes involve drops and there has been growing interest in understanding drop-drop interactions in the presence of electric fields.

A drop placed in an electric field polarizes if its permittivity and/or conductivity are different than the suspending fluid. The polarization leads to a jump in the electric stresses across the drop interface. In the case of fluids that are perfect dielectrics, only the normal electric stress is discontinuous at the interface. If the electric pressure can be

$\dagger$ Email address for correspondence: petia.vlahovska@northwestern.edu 


\section{Sorgentone and others}

balanced by surface tension, the drop adopts a steady prolate ellipsoidal shape and the fluids are quiescent. The physical picture changes dramatically if the fluids are conducting materials. Finite conductivity, even if very low, enables the passage of electric current and electrical charge accumulates at the drop interface. The electric field acting on this induced surface charge creates tangential electric stress, which shears the fluids into motion. The complicated interplay between the electrostatic and viscous fluid stresses results in either oblate or prolate drop deformation in weak fields (Taylor 1966), and a complex dynamics in strong fields, such as break-up (Torza, Cox \& Mason 1971; Sherwood 1988; Lac \& Homsy 2007; Karyappa, Deshmukh \& Thaokar 2014; Lanauze, Walker \& Khair 2015; Pillai et al. 2016; Wang, Ma \& Siegel 2019), streaming either from the drop poles (Taylor 1964; de la Mora 2007; Collins et al. 2008, 2013; Herrada et al. 2012; Sengupta, Walker \& Khair 2017) or equator (Brosseau \& Vlahovska 2017; Wagoner et al. 2020) and electrorotation (Ha \& Yang 2000; Salipante \& Vlahovska 2010, 2013; Das \& Saintillan 2017).

While the prototypical problem of an isolated drop in a uniform electric field has been extensively studied (see for a recent review (Vlahovska 2019)), investigations of the collective dynamics of many drops are scarce (Fernandez 2008a,b; Casas et al. 2019) and mainly focused on the near-contact interaction preceding electrocoalescence (Anand et al. 2019; Roy, Anand \& Thaokar 2019). The dynamics of drop approach and interactions at arbitrary separations has been considered mainly in the case of droplet pairs aligned with the electric field (Sozou 1975; Baygents, Rivette \& Stone 1998; Lin, Skjetne \& Carlson 2012; Mhatre, Deshmukh \& Thaokar 2015; Zabarankin 2020), because the axial symmetry greatly simplifies the calculations. These studies revealed that in weakly conducting fluid systems, which can be modelled using the leaky dielectric model (Melcher \& Taylor 1969), the hydrodynamic interactions due to the electric-shear-driven flow can play a significant role. For example, in the case of a drop with drop-medium ratios of conductivities $R$ and permittivities $S$ such that $R / S>1$, the electrohydrodynamic flow generates repulsion which opposes the electrostatic attraction due to the drop dipoles and the drops move apart.

The general case of an electric field applied at an angle to the line joining the centres of the two drops is studied only to a limited extent experimentally (Mhatre et al. 2015) and via numerical simulations in two dimensions (Dong \& Sau 2018). This configuration has been systematically analysed only for a pair of non-deformable, ideally polarizable spheres (Saintillan 2008). In this case, the flow about the spheres has the same stresslet-quadrupole structure as the electrohydrodynamic flow about a drop with $R / S<1$ even though the flow is due to induced charge electroosmosis, unlike the leaky dielectric drops where Debye charge clouds are absent. The study showed that the pair dynamics is not a simple attraction or repulsion; depending on the angle between the centre-to-centre line and the undisturbed electric field, the relative motion of the two spheres can be quite complex: they attract in the direction of the field and move towards each other, pair up and then separate in the transverse direction. To the best of our knowledge, such a dynamics in the case of drops has not been reported. Motivated by the observed intricate trajectories of ideally polarizable spheres and the potential similarities to the electrohydrodynamic interactions of drops with $R / S<1$, we set out to investigate the effects of drop electric properties (conductivity ratio $R$ and permittivity ratio $S$ ) and deformability on the relative motion of a drop pair initially misaligned with the applied field. The paper is organized as follows: $\S 2$ sets up the problem, $\S 3$ outlines the numerical method, $\S 4$ describes an analytical theory for drop pair interaction and relative motion in an applied uniform DC electric field, $\S 5$ presents results from drop pair dynamics at different initial configurations and drop electrical properties and $\S 6$ summarizes the main results. 


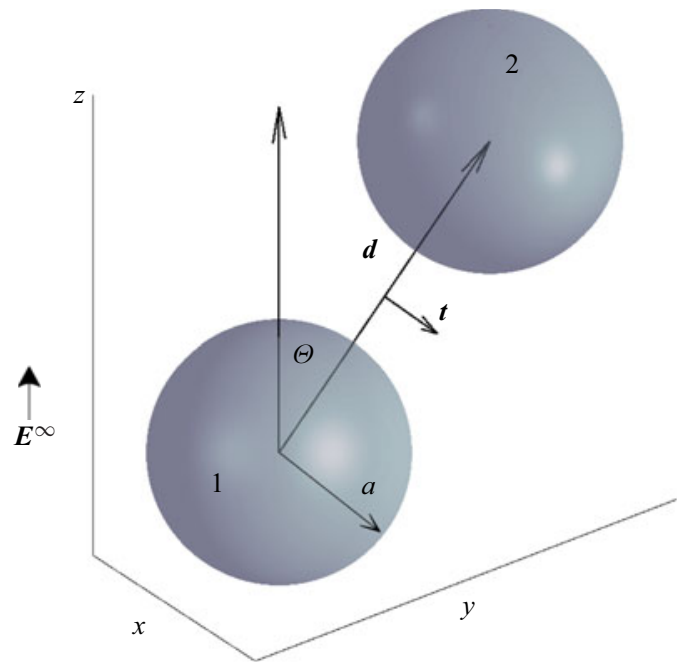

Figure 1. Two initially spherical identical drops with radius $a$, permittivity $\varepsilon_{d}$ and conductivity $\sigma_{d}$ suspended in a fluid with permittivity $\varepsilon_{s}$ and conductivity $\sigma_{s}$ and subjected to a uniform DC electric field $E^{\infty}=E_{0} \hat{z}$. The angle between the line-of-centres vector and the field direction is $\Theta=\arccos (\hat{z} \cdot \hat{d})$.

\section{Problem formulation}

Let us consider two identical neutrally buoyant and charge-free drops with radius $a$, viscosity $\eta_{d}$, conductivity $\sigma_{d}$ and permittivity $\varepsilon_{d}$ suspended in a fluid with viscosity $\eta_{s}$, conductivity $\sigma_{s}$ and permittivity $\varepsilon_{s}$. The mismatch in drop and suspending fluid properties is characterized by the conductivity, permittivity, and viscosity ratios

$$
R=\frac{\sigma_{d}}{\sigma_{s}}, \quad S=\frac{\varepsilon_{d}}{\varepsilon_{s}}, \quad \lambda=\frac{\eta_{d}}{\eta_{s}} .
$$

The distance between the drops' centroids is $d$ and the angle made with the drops' line of centres and the applied field direction is $\Theta$. The unit separation vector between the drops is defined by the difference between the position vectors of the drops' centres of mass $\hat{d}=\left(x_{2}^{c}-x_{1}^{c}\right) / d$. The unit vector normal to the drops' line of centres and orthogonal to $\hat{d}$ is $\hat{t}$. The problem geometry is sketched in figure 1 .

We adopt the leaky dielectric model (Melcher \& Taylor 1969), which assumes creeping flow and charge-free bulk fluids acting as ohmic conductors. Albeit an approximation of the actual electrokinetic problem (Saville 1997; Schnitzer \& Yariv 2015; Ganan-Calvo et al. 2016, 2018; Mori \& Young 2018), the leaky dielectric model has been successful in modelling many phenomena not only in poorly conducting fluids such as oils, but also aqueous electrolyte solutions such as in cell-mimicking vesicle systems (Vlahovska et al. 2009; Vlahovska 2019). The assumption of charge-free fluids decouples the electric and hydrodynamic fields in the bulk. Accordingly,

$$
\nabla \cdot T^{h d}=\eta \nabla^{2} u-\nabla p=0, \quad \nabla \cdot T^{e l}=0,
$$

where $T_{i j}^{h d}=-p \delta_{i j}+\eta\left(\partial_{j} u_{i}+\partial_{i} u_{j}\right)$ is the hydrodynamic stress and $\delta_{i j}$ is the Kronecker delta function; $u$ and $p$ are the fluid velocity and pressure. The electric stress is given by the Maxwell stress tensor $T_{i j}^{e l}=\varepsilon\left(E_{i} E_{j}-E_{k} E_{k} \delta_{i j} / 2\right)$. The coupling of the electric field and the fluid flow occurs at the drop interfaces $\mathcal{D}$, where the charges 


\section{Sorgentone and others}

brought by conduction accumulate. Gauss' law dictates that the electric field $E$ in the electroneutral bulk fluids is solenoidal, $\nabla \cdot E=0$, however, at the drop interface, the electric displacement field, $\varepsilon \boldsymbol{E}$, is discontinuous and its jump corresponds to the surface charge density

$$
\varepsilon\left(E_{n}^{s}-S E_{n}^{d}\right)=q, \quad x \in \mathcal{D},
$$

where $E_{n}=\boldsymbol{E} \cdot \boldsymbol{n}$, and $\boldsymbol{n}$ is the outward pointing normal vector to the drop interface. The surface charge density adjusts to satisfy the current balance

$$
\frac{\partial q}{\partial t}+\nabla_{s} \cdot(\boldsymbol{u} q)=\sigma_{s}\left(E_{n}^{s}-R E_{n}^{d}\right), \quad \boldsymbol{x} \in \mathcal{D} .
$$

In this study, we neglect charge relaxation and convection, thereby reducing the charge conservation equation to continuity of the electrical current across the interface as originally proposed by Taylor (1966)

$$
E_{n}^{s}=R E_{n}^{d}
$$

The electric field acting on the induced surface charge gives rise to an electric shear stress at the interface. The tangential stress balance yields

$$
(I-n n) \cdot\left(T^{s}-T^{d}\right) \cdot n+q E_{t}=0, \quad x \in \mathcal{D},
$$

where $E_{t}=E-E_{n} \boldsymbol{n}$ is the tangential component of the electric field, which is continuous across the interface, and $\boldsymbol{I}$ is the idemfactor. The normal stress balance is

$$
\boldsymbol{n} \cdot\left(\boldsymbol{T}^{S}-\boldsymbol{T}^{d}\right)+\frac{1}{2}\left(\left(E_{n}^{s}\right)^{2}-S\left(E_{n}^{d}\right)^{2}-(1-S) E_{t}^{2}\right)=\gamma\left(\nabla_{s} \cdot \boldsymbol{n}\right) \boldsymbol{n}, \quad \boldsymbol{x} \in \mathcal{D},
$$

where $\gamma$ is the interfacial tension.

Henceforth, all variables are non-dimensionalized using the radius of the undeformed drops $a$, the undisturbed field strength $E_{0}$, a characteristic applied stress $\tau_{c}=\varepsilon_{s} E_{0}^{2}$ and the properties of the suspending fluid. Accordingly, the time scale is $t_{c}=\eta_{s} / \tau_{c}$ and the velocity scale is $u_{c}=a \tau_{c} / \eta_{s}$. The ratio of the magnitude of the electric stresses and surface tension defines the electric capillary number

$$
C a=\frac{\varepsilon_{s} E_{0}^{2} a}{\gamma} .
$$

The simplification of the charge conservation equations (2.4) and (2.5) implies $\varepsilon_{s}^{2} E_{0}^{2} /\left(\eta_{s} \sigma_{s}\right) \ll 1$. This condition is satisfied for the typical fluids used in experiments such as castor oil (conductivity is $\sim 10^{-11} \mathrm{~S} \mathrm{~m}^{-1}$, viscosity is $\sim 1 \mathrm{~Pa} \mathrm{~s}$ ) and low field strengths $E_{0} \sim 10^{4} \mathrm{~V} \mathrm{~m}^{-1}$. Furthermore, the momentum diffusion time scale, $a^{2} \rho / \eta_{s}$, for drops of typical size $a \sim 1 \mathrm{~mm}$ is much shorter than the electrohydrodynamic flow time scale $\eta_{s} /\left(\varepsilon_{s} E_{0}^{2}\right)$, which justifies the use of the steady Stokes equation to describe the fluid flow (2.2).

\section{Numerical method}

We utilize the boundary integral method to solve for the flow and electric fields. Details of our three-dimensional formulation can be found in Sorgentone, 
Tornberg \& Vlahovska (2019). In brief, the electric field is computed following (Baygents et al. 1998; Lac \& Homsy 2007)

$$
\boldsymbol{E}^{\infty}+\sum_{j=1}^{2} \int_{\mathcal{D}_{j}} \frac{\hat{\boldsymbol{x}}}{4 \pi r^{3}}\left(\boldsymbol{E}^{s}-\boldsymbol{E}^{d}\right) \cdot \boldsymbol{n} \mathrm{d} S(\boldsymbol{y})= \begin{cases}\boldsymbol{E}^{d}(\boldsymbol{x}) & \text { if } \boldsymbol{x} \text { inside } \mathcal{D} \\ \frac{1}{2}\left(\boldsymbol{E}^{d}(\boldsymbol{x})+\boldsymbol{E}^{s}(\boldsymbol{x})\right) & \text { if } \boldsymbol{x} \in \mathcal{D} \\ \boldsymbol{E}^{s}(\boldsymbol{x}) & \text { if } \boldsymbol{x} \text { outside } \mathcal{D}\end{cases}
$$

where $\hat{\boldsymbol{x}}=\boldsymbol{x}-\boldsymbol{y}$ and $r=|\hat{\boldsymbol{x}}|$. The normal and tangential components of the electric field are calculated from the above equation

$$
\left.\begin{array}{c}
E_{n}(\boldsymbol{x})=\frac{2 R}{R+1} \boldsymbol{E}^{\infty} \cdot \boldsymbol{n}+\frac{R-1}{R+1} \sum_{j=1}^{2} \boldsymbol{n}(\boldsymbol{x}) \cdot \int_{\mathcal{D}_{j}} \frac{\hat{\boldsymbol{x}}}{2 \pi r^{3}} E_{n}(\boldsymbol{y}) \mathrm{d} S(\boldsymbol{y}), \\
\boldsymbol{E}_{t}(\boldsymbol{x})=\frac{\boldsymbol{E}^{S}+\boldsymbol{E}^{d}}{2}-\frac{1+R}{2 R} E_{n} \boldsymbol{n} .
\end{array}\right\}
$$

For the flow field, we have developed the method for fluids of arbitrary viscosity, but for the sake of brevity here we list the equation in the case of equiviscous drops and suspending fluids. The velocity is given by

$$
2 \boldsymbol{u}(\boldsymbol{x})=-\sum_{j=1}^{2}\left(\frac{1}{4 \pi} \int_{\mathcal{D}_{j}}\left(\frac{\boldsymbol{f}(\boldsymbol{y})}{C a}-f^{E}(\boldsymbol{y})\right) \cdot\left(\frac{\boldsymbol{I}}{r}+\frac{\hat{\boldsymbol{x}} \hat{\boldsymbol{x}}}{r^{3}}\right) \mathrm{d} S(\boldsymbol{y})\right)
$$

where $f$ and $f^{E}$ are the interfacial stresses due to surface tension and electric field

$$
\boldsymbol{f}=\boldsymbol{n} \nabla_{s} \cdot \boldsymbol{n}, \quad \boldsymbol{f}^{E}=\left(\boldsymbol{E}^{s} \cdot \boldsymbol{n}\right) \boldsymbol{E}^{s}-\frac{1}{2}\left(\boldsymbol{E}^{s} \cdot \boldsymbol{E}^{s}\right) \boldsymbol{n}-S\left(\left(\boldsymbol{E}^{d} \cdot \boldsymbol{n}\right) \boldsymbol{E}^{d}-\frac{1}{2}\left(\boldsymbol{E}^{d} \cdot \boldsymbol{E}^{d}\right) \boldsymbol{n}\right) .
$$

Drop velocity and centroid are computed from the volume averages

$$
\boldsymbol{U}_{j}=\frac{1}{V} \int_{V_{j}} \boldsymbol{u} \mathrm{d} V=\frac{1}{V} \int_{\mathcal{D}_{j}} \boldsymbol{n} \cdot(\boldsymbol{u} \boldsymbol{x}) \mathrm{d} S, \quad \boldsymbol{x}_{j}^{c}=\frac{1}{V} \int_{V_{j}} \boldsymbol{x} \mathrm{d} V=\frac{1}{2 V} \int_{\mathcal{D}_{j}} \boldsymbol{n}(\boldsymbol{x} \cdot \boldsymbol{x}) \mathrm{d} S .
$$

To solve the system of (3.2) and (3.3) we utilize the boundary integral method presented in Sorgentone et al. (2019). In the current study, however, we modify the time-stepper scheme to the adaptive fourth-order Runge-Kutta introduced in Kennedy \& Carpenter (2003). All variables are expanded in spherical harmonics, which provides an accurate representation even for relatively low expansion order. In this respect, to make sure that all the geometrical quantities of interest (e.g. mean curvature) are computed with high accuracy as well, we adopt an adaptive upsampling procedure introduced by Rahimian et al. (2015) which is based on the decay of the mean curvature spectrum and seems to work very well for this kind of simulation. When the drops are well separated from each other, the regular quadrature based on the trapezoidal rule in the longitudinal direction and on the Gauss-Legendre quadrature rule in the non-periodic direction works well. As they get closer, regular quadrature on a finer grid can still be used. Here, the density is interpolated to the finer (upsampled) grid, where the nearly singular kernel is better resolved. But at some point, a special quadrature method is needed since the quadrature error grows exponentially as we approach the surface and it is not possible to resolve the problem by grid refinement, i.e. upsampling. In Sorgentone \& Tornberg (2018) a 


\section{Sorgentone and others}

numerical procedure based on interpolation first introduced by Ying, Biros \& Zorin (2006) is discussed and optimized to handle these complicated situations. The idea introduced in Ying et al. (2006) for the nearly singular integration was to find the point $x_{*}$ on the surface that is closest to the target point $x_{0}$. Then, continuing along a line that passes through $x_{*}$ and $x_{0}$, the integral is evaluated at a number of points $x_{1}, \ldots, x_{n}$ further away from the surface. This can be done by regular quadrature on the standard grid or on the upsampled grid, depending on how far the target point is from the surface. The value of the integral on the surface (at $x_{*}$ ) needs to be computed by a specialized quadrature rule for singular integrals. At this point a one-dimensional Lagrangian interpolation is used to compute the value at $x_{0}$ by interpolating the values at $x_{*}$ and $x_{i}, i=1, \ldots, N$. In Sorgentone $\&$ Tornberg (2018) it has been shown how to optimize this procedure, implementing a cell list algorithm to hierarchically find the closest point on the surface and using the spherical harmonic expansion to interpolate the on-surface integral value previously obtained on the whole surface (at the grid points only) by the special quadrature for singular integrals introduced in Veerapaneni et al. (2011) and Rahimian et al. (2015). The accuracy of the method depends on the numerical parameters involved: the maximum distance before we need to upsample the grid for the regular quadrature (that of course will depend on the grid resolution), the upsampling rate used in the intermediate region, the number of points used for interpolation for target points in the nearest region, the distance and the distribution of these points (Sorgentone \& Tornberg 2018). We also use the spectral reparameterization technique presented in the same paper, designed to keep the representation optimal even under strong deformations. In our work, in order to be able to run long simulations and well resolve the close interactions, we set the spherical harmonic expansion order to $p=9$, and for the nearly singular quadrature, we set the upsampling rate in the intermediate region to 4 and the number of interpolating points to 8 . The viscosity contrast is $\lambda=1$. Unless otherwise explicitly stated, the electric capillary number is $C a=0.1$.

Our numerical method was validated against the simulation results of Baygents et al. (1998) and an analytical theory for spherical drops developed by us and presented in the next section. Figure 2 shows the results for the drop steady velocity as a function of the drop centroid separation for drops aligned with the field. Figure 3 illustrates the more general case of drops initially misaligned with the field. The simulations agree very well with the theory and show a complex dynamics such as the drops line-of-centres rotating away from the applied field direction and interaction switching from attraction to repulsion. This dynamics will be explored in more detail in $\S 5$.

\section{Theory: far-field interactions}

To gain more physical insight, it is instructive to analyse the interaction of two widely separated spherical drops. In this case, the drops can be approximated as point dipoles. The disturbance field $\boldsymbol{E}_{1}$ of the drop dipole $\boldsymbol{P}_{1}$ induces a dielectrophoretic (DEP) force on the dipole $\boldsymbol{P}_{2}$ located at $\boldsymbol{x}_{2}^{c}=d \hat{\boldsymbol{d}}$, given by $\boldsymbol{F}(d)=\left.\left(\boldsymbol{P}_{2} \cdot \nabla \boldsymbol{E}_{1}\right)\right|_{r=d}$

$$
\boldsymbol{F}(d)=\boldsymbol{P}_{1} \boldsymbol{P}_{2}:\left.\nabla\left(\frac{\boldsymbol{I}}{r^{3}}-3 \frac{\boldsymbol{x} \boldsymbol{x}}{r^{5}}\right)\right|_{r=d}, \quad \boldsymbol{P}_{1}=\boldsymbol{P}_{2}=\frac{R-1}{R+2} E^{\infty} .
$$

The drop velocity under the action of this force can be estimated from Stokes' law, $\boldsymbol{U}_{2}^{\text {dep }}=$ $-U_{1}^{d e p}=F / \zeta$, where $\zeta$ is the friction coefficient, $\zeta=2 \pi(3 \lambda+2) /(\lambda+1)$

$$
U_{2}^{d e p}=\frac{\beta_{D}}{r^{4}} \frac{3(1+\lambda)}{2+3 \lambda}\left[\left(1-3 \cos ^{2} \Theta\right) \hat{d}-\sin (2 \Theta) \hat{\boldsymbol{t}}\right], \quad \beta_{D}=\left(\frac{R-1}{R+2}\right)^{2} .
$$




\section{Electrohydrodynamic interaction of drop pairs}
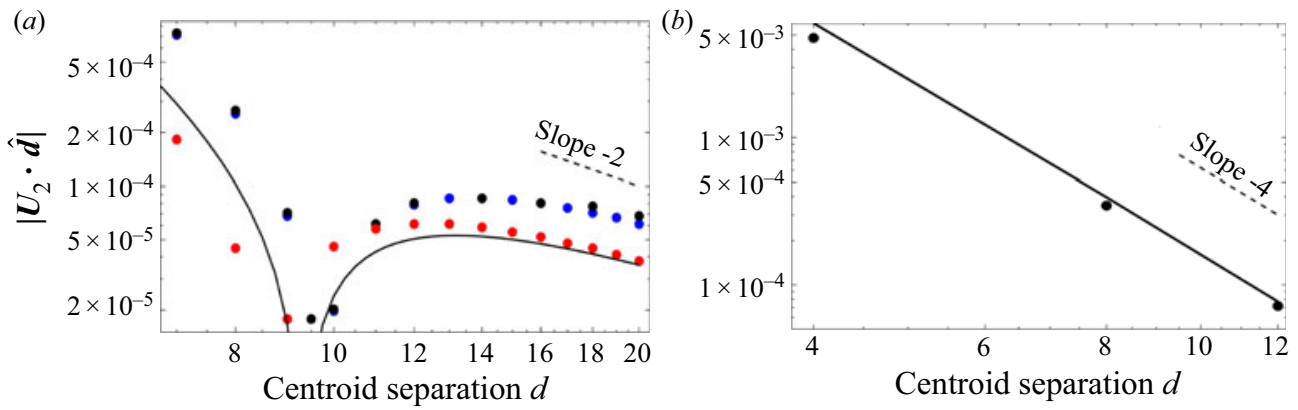

Figure 2. Comparison between our fully three-dimensional simulations and the axisymmetric simulations of a drop pair aligned with the field by Baygents et al. (1998). (a) Absolute value of the radial component of the steady drop velocity as a function of separation for $C a=0.1$ (red dots) and 1 (blue dots) for a drop with $R=5$, $S=4$. Black dots are the data from figure 9 in Baygents et al. (1998) with $\mathrm{Ca}=1$. Solid line is the theoretical $U_{2} \cdot \hat{d}$ for a drop of low $\mathrm{Ca}$ given by (4.7). The drop velocity at large separations shows the $1 / d^{2}$ behaviour of a stresslet flow. (b) Absolute value of the steady velocity of a drop undergoing only a dielectrophoretic force, $R=S=5$, corresponding to figure 4(a) in Baygents et al. (1998). The black dots are from our fully three-dimensional code, the solid line is the dielectrophoretic velocity, (4.2), showing $1 / d^{4}$ dependence.
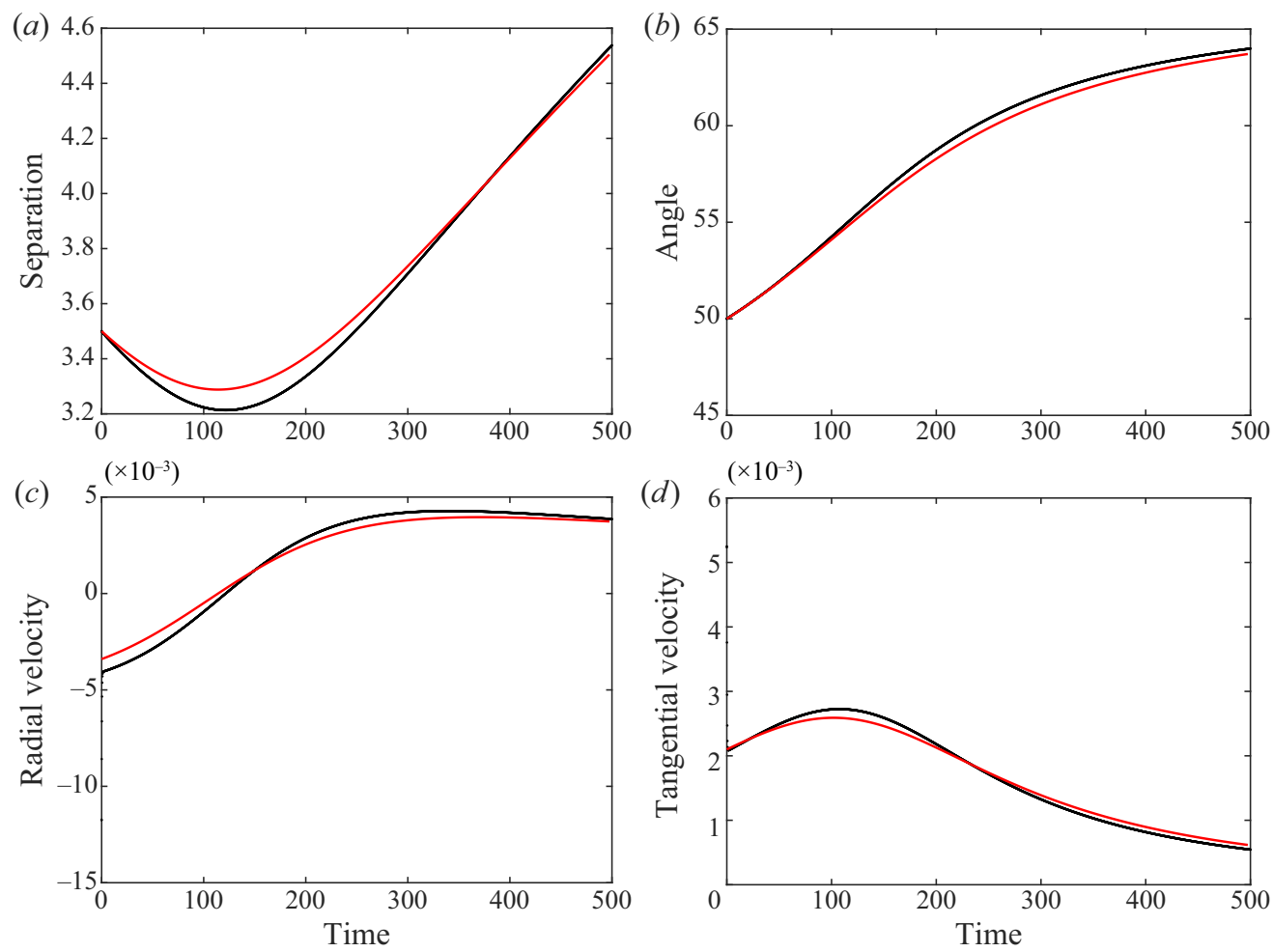

Figure 3. Comparison between the simulations (black) and the analytical theory (red) for a drop pair with $R=1, S=3$, initial separation 3.5, initial angle between the line of centres and the applied field direction $50^{\circ}$ and $\mathrm{Ca}=0.1$. The trajectory was computed from the relative drop velocity (4.7). Time evolution of the (a) separation, (b) angle between the line of centres and applied field direction, $(c)$ radial component of the relative velocity $U \cdot \hat{d}$ and $(d)$ tangential component of the relative velocity $U \cdot \hat{t}$. 


\section{Sorgentone and others}

The relative DEP velocity, $U^{d e p}=U_{2}^{d e p}-U_{1}^{d e p}$, depends on the angle $\Theta=\arccos (\hat{\boldsymbol{d}} \cdot \hat{z})$ between the direction of the external field and the line joining the centres of the two drops. Drops attract, i.e. $U^{d e p} \cdot \hat{d}<0$, if $\Theta<\Theta_{c}=\arccos (1 / \sqrt{3}) \approx 54.7^{\circ}$, e.g. when the drops are lined up with the field, and repulse if $\Theta>\Theta_{c}$, e.g. if the line of centres of the two drops is perpendicular to the field. The DEP interaction also causes drops to align with the field, since the tangential component of the relative velocity is always negative.

The electrohydrodynamic (EHD) flow about an isolated, spherical drop in an applied uniform electric field is a combination of a stresslet and a quadrupole (see appendix A for the flow evolution upon application of the electric field). At steady state,

$$
u=\frac{9}{10} \frac{S-R}{(2+R)^{2}(\lambda+1)} E^{\infty} E^{\infty}:\left[\left(\frac{I}{r^{3}}-3 \frac{x \boldsymbol{x}}{r^{5}}\right) x+\frac{1}{3} \nabla\left(\frac{I}{r^{3}}-3 \frac{x \boldsymbol{x}}{r^{5}}\right)\right] .
$$

At the surface of the drop,

$$
\boldsymbol{u}(r=1)=\beta_{T} \sin (2 \theta) \hat{\boldsymbol{\theta}}, \quad \beta_{T}=\frac{9}{10} \frac{R-S}{(1+\lambda)(R+2)^{2}} .
$$

If $R / S<1$, the surface flow is from pole to equator, i.e. fluid is drawn in at the poles and pushed away from the drop at the equator. The flow direction is reversed for $R / S>1$. A second drop moves in response to the EHD flow (4.3). The drop translational velocity is found from Faxen's law (Kim \& Karrila 1991)

$$
\boldsymbol{U}_{2}^{\text {ehd }}=\left.\left(1+\frac{\lambda}{2(3 \lambda+2)} \nabla^{2}\right) \boldsymbol{u}\right|_{x=d \hat{d}} .
$$

Inserting (4.3) in the above equation leads to

$$
\begin{aligned}
U_{2}^{e h d}= & \beta_{T}\left(\frac{1}{d^{2}}-\frac{2}{d^{4}}\left(\frac{1+3 \lambda}{2+3 \lambda}\right)\right)\left(-1+3 \cos ^{2} \Theta\right) \hat{\boldsymbol{d}} \\
& -\frac{2 \beta_{T}}{d^{4}}\left(\frac{1+3 \lambda}{2+3 \lambda}\right) \sin (2 \Theta) \hat{\boldsymbol{t}}+O\left(d^{-5}\right) .
\end{aligned}
$$

The radial component of the EHD velocity, and thus the sign of the EHD interaction (attraction or repulsion), changes sign at the same angle as the DEP interaction, $\Theta_{c}=54.7$. If $R / S<1$, the EHD flow is attractive when drops are aligned with the applied field and repulsive when the line of centres is perpendicular to the field direction. The interaction is reversed for $R / S>1$. Notably, unlike the DEP interaction which always drives the drops to align with the applied field direction, the EHD can cause the drops' line of centres to rotate away from the direction of the applied field if $\beta_{T}<0$, i.e. $R / S<1$.

Combining the EHD and the DEP velocities yields the relative drop velocity. Since the drops studied here are identical, drop motions are reciprocal. Therefore, the relative drop velocity $U=U_{2}-U_{1}=2 U_{2}$

$$
U=\frac{2 \beta_{T}}{d^{2}}\left(-1+3 \cos ^{2} \Theta\right) \hat{\boldsymbol{d}}-\Phi(R, S, \lambda) \frac{4}{d^{4}}\left(\left(-1+3 \cos ^{2} \Theta\right) \hat{\boldsymbol{d}}+\sin (2 \Theta) \hat{\boldsymbol{t}}\right)+O\left(d^{-5}\right),
$$

where

$$
\Phi=\frac{1+3 \lambda}{2+3 \lambda}\left(\beta_{T}+3 \beta_{D} \frac{1+\lambda}{1+3 \lambda}\right) .
$$

The discriminant $\Phi$ quantifies the drop pair alignment with the field and the interplay of EHD and DEP interactions in drop attraction or repulsion. The line of centres between 

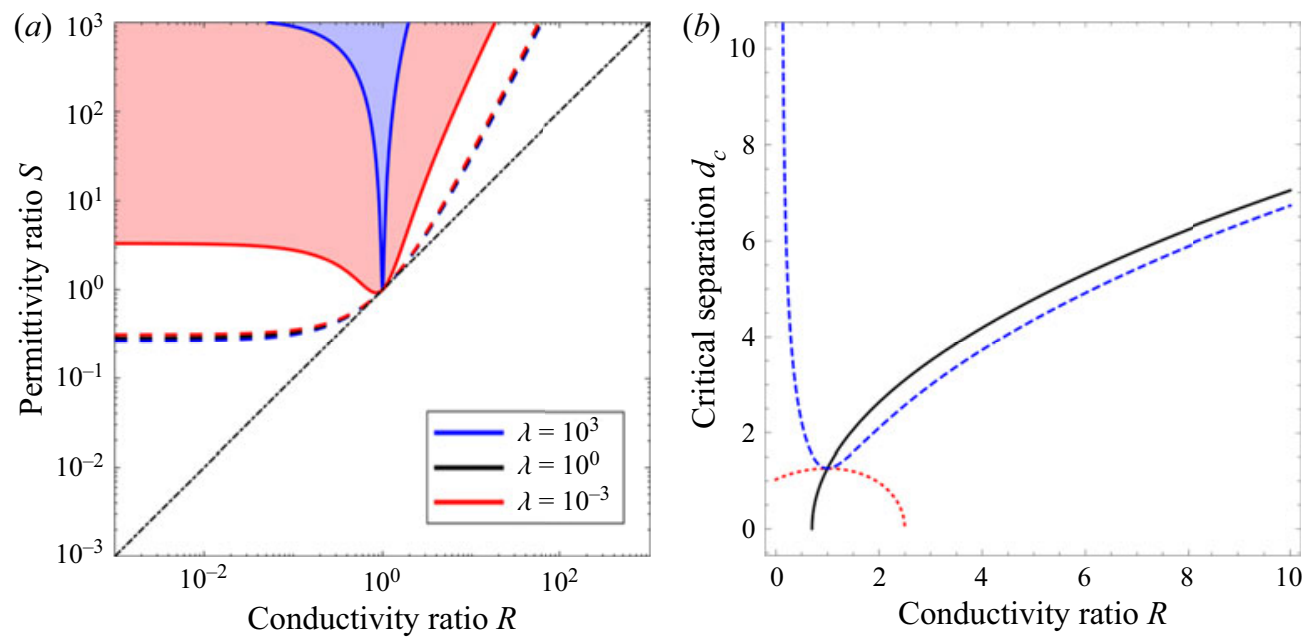

Figure 4. (a) Phase diagram of drop deformations and alignment with the field for different viscosity ratios. The solid lines correspond to $\Phi(\lambda, R, S)=0$ given by (4.8). In the shaded regions, the line of centres of the two drops rotates away from the applied field direction $\Phi<0$. The dashed line corresponds to the Taylor discriminating function (A3); in the parameter space above it, drop deformation is oblate. (b) Critical separation above which EHD dominates the interactions for $\lambda=1$ and $S=0.1$ (blue), $S=1$ (black) and $S=10$ (red).

two drops with $\Phi>0$ rotates towards a parallel orientation with respect to the applied electric field, since $\dot{\Theta}=U \cdot \hat{\boldsymbol{t}} \sim-\Phi$. However, in the case of $\Phi<0$ (which occurs only for $R / S<1$ drops), the line of centres between the drops rotates towards a perpendicular orientation with respect to the applied electric field. Figure 4(a) summarizes the regimes of alignment and deformation.

The relative radial motion of the two drops at a given configuration depends on $\Phi$ and $\beta_{T}$, where $\beta_{T} \hat{z} \hat{z}$ is the strength of the far-field EHD stresslet flow

$$
\boldsymbol{u}_{s}(\boldsymbol{x})=\beta_{T}\left(-1+3 \cos ^{2} \theta\right) \frac{\boldsymbol{x}}{r^{3}} .
$$

There is a critical separation $d_{c}$ corresponding to $U_{2}\left(d_{c}\right) \cdot \hat{\boldsymbol{d}}=0$, which yields $d_{c}^{2}=$ $2 \Phi / \beta_{T}$. For $\Phi>0$ and $R / S<1\left(\beta_{T}<0\right), d_{c}$ does not exist and EHD and DEP interactions are cooperative and act radially in the same direction (note that a system with $\Phi<0$ and $R / S>1$ cannot exist). For $\Phi>0$ and $R / S>1$ or $\Phi<0$ and $R / S<1$, there is competition between EHD and DEP, with the quadrupolar DEP winning out closer to the drops and the EHD taking over via the stresslet flow in the far field. The fact that depending on separation drops may attract or repel in the case of antagonistic EHD and DEP interactions has been discussed previously by Baygents et al. (1998) and Zabarankin (2020). Note that for drops with $R / S<1$, EHD effectively dominates DEP at all separations since $d_{c}$ is smaller than 2 , which is the minimum separation of spherical drops. Figure $4(b)$ illustrates the dependence of the critical separation $d_{c}$ for three typical cases. If $S=10, d_{c}$ is always less than 2 , the minimum separation between two spheres, and accordingly the interactions are dominated by the EHD flow. For $S=1, d_{c}$ does not exist below $R=0.7$. In the case $S=0.1, \Phi>0$ for all values of $R$ and thus $R / S<1$ is always dominated by EHD, while in the case $R / S>1$ the DEP attraction could be stronger than the EHD for quite large separations, e.g. for $R / S=1.1$ or $R / S \gg 1 d_{c}>10$. The DEP dominates in these cases because the EHD is very weak, in the first case because 


\section{Sorgentone and others}

(a)

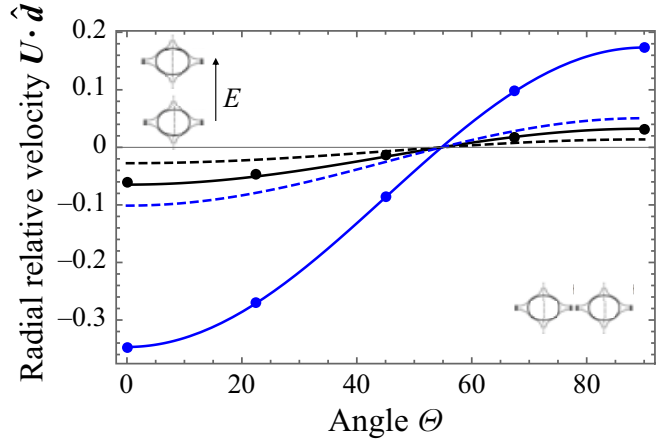

(b)

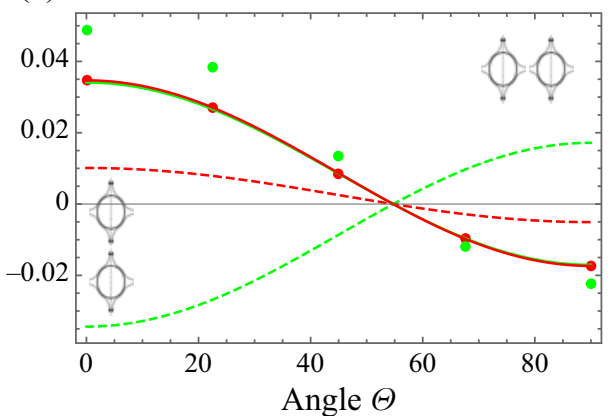

Figure 5. Radial component $U \cdot \hat{d}$ of the relative velocity of the two drops $U$ at $t=0$ as a function of the angle made between the applied field and the line of centres of the two spheres $E^{\infty} \cdot \hat{d}=\cos \Theta$. Initial separation is $d=4$. (a) Case $R / S<1: R=0.1, S=1$ (black), $R=1, S=10$ (blue). At $\Theta=0$, both electrostatic (DEP) and EHD interactions are attractive. At $\Theta=\pi / 2$, both DEP and EHD are repulsive. (b) Case $R / S>1: R=$ $1, S=0.1$ (red), $R=100, S=1$ (green). Solid line corresponds to the velocity computed from the theory accounting for transient drop deformation (A9), while the dashed line corresponds to the theory assuming spherical drops (4.7). Points are the numerical simulations.

$(R-S) \sim 0$ and in the second case because the EHD flow decreases with conductivity ratio as $\sim 1 / R$.

\section{Results and discussion}

An isolated charge-neutral drop in a uniform DC electric field experiences no net force. However, a drop pair can move in response to mutual electrostatic (due to polarization) and hydrodynamic (due to the flow driven by surface electric stresses) interactions. While the theory in $\S 4$ describes steady drop velocities (drop shape is assumed to be spherical and unaffected by drop motions), our simulations consider deformable drops whose shape can change during the interaction. We have extended the quasi-steady theory to account for the contribution of transient small deformations in the EHD drop velocity, see appendix A for details. Here, we explore the pair dynamics at different initial configurations using the simulations, the quasi-steady and the transient-deformation theories.

\subsection{Initial drop interactions}

The initial interaction of two drops at different misalignment with the applied field is illustrated in figures 5 and 6 by the dependence on $\Theta$ of the initial $(t=0)$ relative velocity of the two drops. Figure 5 shows that the radial component of the velocity changes sign around $\Theta \sim \Theta_{c}$. The critical angle at which the total interaction changes sign at different separations between the drops is shown in figure $6(b)$. The deviation from the far-field result $\Theta_{c}=54.7^{\circ}$ is due to the DEP interaction, since the dipole approximation becomes inaccurate at small separations. The value $R=1$ turns off the DEP interaction and in this case the angle is exactly given by $\Theta_{c}=54.7^{\circ}$ at all separations. This is because the EHD solution (4.5) is exact for a sphere, which is the drop shape at $t=0$.

In the case $R / S<1$, the centre-to-centre electrostatic (DEP) and EHD interactions work in the same direction. Figure $5(a)$ shows that when the drop pair is aligned with the field $(\Theta=0)$, the drops attract. As $\Theta$ increases, the attraction decreases and changes to repulsion around $\Theta \sim \Theta_{c}$. The repulsion is strongest in the configuration with $\Theta=\pi / 2$, i.e. line of drop centres perpendicular to the applied field. The initial velocity is higher 

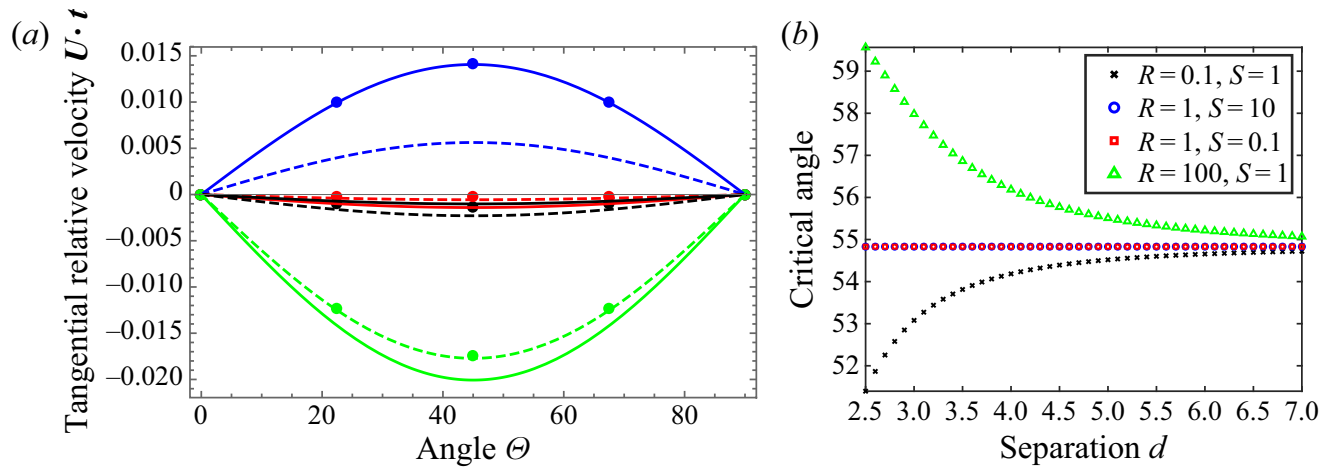

Figure 6. (a) Tangential component $U \cdot \hat{t}$ of the relative velocity of the two drops $U$ at $t=0$ as a function of the angle made between the applied field and the line of centres of the two drops $E^{\infty} \cdot \hat{d}=\cos \Theta$. Initial separation is $d=4 . R=1, S=10$ (blue), $R=0.1, S=1$ (black), $R=1, S=0.1$ (red), $R=100$, $S=1$ (green). Solid line corresponds to the velocity computed from the theory accounting for transient drop deformation (A9), while the dashed line corresponds to the theory assuming spherical drops (4.7). Points are the numerical simulations. (b) Critical value of the angle $\Theta$ for which the initial radial velocity between two spheres is zero, plotted as a function of the separation distance between the drops. This critical angle separates configurations for which drops attract $(\boldsymbol{U} \cdot \hat{d}<0)$ and repel $(\boldsymbol{U} \cdot \hat{d}>0)$. Points are the numerical simulations and the lines are added to guide the eye.

than predicted by the theory assuming spherical drops, because at $t=0$, the drop shape begins to evolve and thus the fluid around the drop moves not only because of the tangential electric shearing of the interface, but also because the interface deforms. As a result, the strength of the EHD contribution to the relative velocity at early times is enhanced.

The case $R / S>1$ is more complicated because the electrostatic and EHD interactions are antagonistic. The EHD interactions are predicted to change from repulsive to attractive as $\Theta$ increases, while the DEP follows the opposite trend. Figure 5(b) shows that the interaction at $t=0$ for the considered separation $d=4$ is dominated by the EHD contribution. The theory assuming spherical drops predicts that DEP dominates the interaction of the drops with $R=100, S=1$, since for this system the critical separation $d_{c}=\sqrt{2 \Phi / \beta_{T}} \sim 23$ is much larger than the initial separation. However, at $t=0$ the flow associated with the elongating drops overcomes the DEP. The solution of the transient EHD problem, which accounts for the drop shape evolution (see appendix A), does highlight that the relative velocity can reverse sign before deformation reaches steady state on a typical time scale $\sim \mathrm{Ca}$.

The tangential component of the relative velocity is $\boldsymbol{U} \cdot \hat{\boldsymbol{t}}=-4 \Phi \sin (2 \Theta) / d^{4}$. Accordingly, it is maximal at $\Theta=\pi / 4$ as confirmed by figure 6(a). In all cases except $R=1, S=10$ the tangential velocity is negative, indicating that the drops' line of centres will move towards the applied field direction.

The question arises as to what happens after the initial attraction or repulsion? How do drop deformation, hydrodynamic and electrostatic interactions affect the drop trajectory? Here we show that their interplay gives rise to intricate trajectories.

\subsection{Drop pair trajectories: evolution of separation and alignment with the field}

Figures 7 and 8 illustrate the evolution of the centre-to-centre distance, the radial component of the relative velocity and the deformation parameter of drop 1 in the case of drops initially placed in the two extreme configurations, aligned and perpendicular to the field, $\Theta=0$ and $\Theta=\pi / 2$, respectively. The simulations are compared to the 


\section{Sorgentone and others}
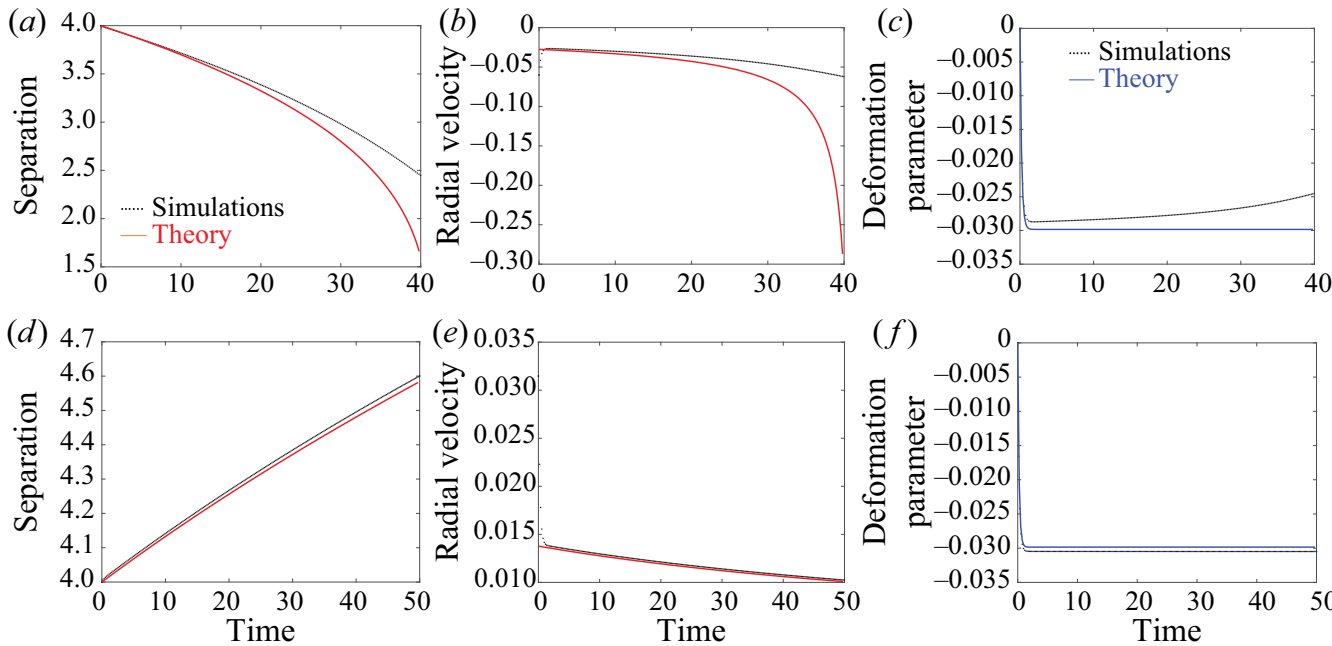

Figure 7. The EHDs of a pair of deforming drops with $R=0.1$ and $S=1$ with initial separation $d=4$ and inclinations $\Theta=0(a-c)$ and $\Theta=\pi / 2(d-f)$. Evolution of the centre-to-centre distance $(a, d)$, the radial component of the relative velocity $(b, e)$ and the deformation parameter $(c, f)$. Black dots correspond to the numerical simulations. Red line corresponds to the radial velocity and separation predicted by (4.7) and the blue line is the deformation of an isolated drop calculated from (5.2).

quasi-steady theory, where the separation is computed from the radial velocity, $\dot{d}=\boldsymbol{U} \cdot \hat{d}$ with $U$ given by (4.7). The tangential component of the relative velocity, $\boldsymbol{U} \cdot \hat{\boldsymbol{t}}$, is zero during the interaction and accordingly the drop pair orientation with the field remains unchanged, i.e. the angle between the line of centres (and the field direction) remains in the initial configuration. In both cases, $R / S<1$ and $R / S>1$, the drops attract in the $\Theta=0$ configuration, and repel if aligned perpendicularly with the applied field, $\Theta=\pi / 2$. However, the interaction in the $R / S<1$ case is controlled by EHD, while in the $R / S>1$ case - by DEP, since the critical distance $d_{c}$ in the considered system $R=100, S=1$ is approximately 23 , much larger than the initial separation. The radial velocity, $U \cdot \hat{d}$, varies in time and in the case $R / S<1$ (figure 7) does not change sign (it remains either negative, indicating attraction, or positive, indicating repulsion). In the $\Theta=0$ case, drops attract and the distance between the drops decreases; if $\Theta=\pi / 2$, the drops repel and the separation increases. In the case $R / S>1$ (figure 8 ), the radial velocity reverses sign on a short time scale $\sim C a$. If $\Theta=0$, drops attract after a short transient repulsion and separation decreases in time. The opposite occurs in the $\Theta=\pi / 2$ configuration. The theoretical trajectory computed from the steady state velocity and the simulations are in good agreement since drop shape remains close to a sphere and drop translation is slow compared to the deformation time scale.

The deformation parameter is defined as $D_{T}=\left(a_{\|}-a_{\perp}\right) /\left(a_{\|}+a_{\perp}\right)$, where $a_{\|}$and $a_{\perp}$ are the drop lengths in directions parallel and perpendicular to the applied field. For an isolated drop, in weak fields $(\mathrm{Ca} \ll 1)$ the equilibrium shape is given by

$$
D_{T}=\frac{9 C a}{16(2+R)^{2}}\left[R^{2}+1-2 S+3(R-S) \frac{2+3 \lambda}{5(1+\lambda)}\right] .
$$

Upon application of the field, the drop approaches the steady state monotonically (Esmaeeli \& Sharifi 2011)

$$
D(t)=D_{T}\left(1-e^{-t / t_{r}}\right) \quad \text { where } t_{r}=\frac{\eta_{s} a}{\gamma}\left(\frac{(3+2 \lambda)(16+19 \lambda)}{40(1+\lambda)}\right) .
$$



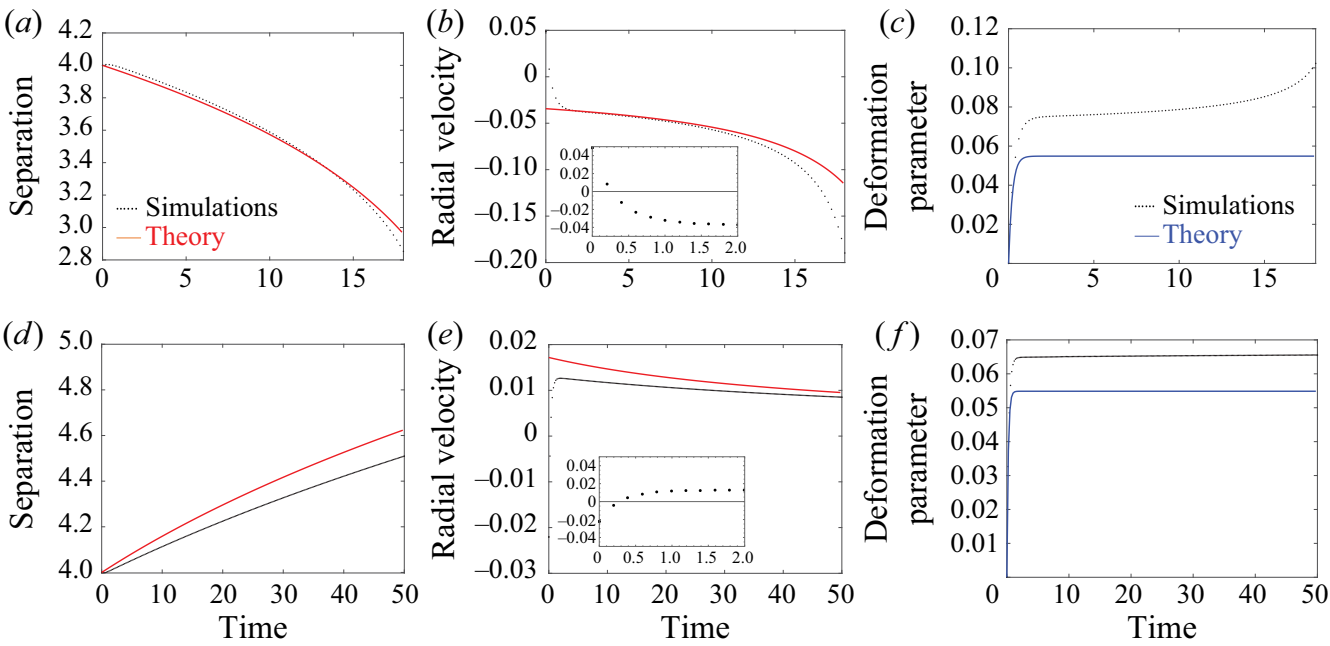

Figure 8. The EHDs of a pair of deforming drops with $R=100$ and $S=1$ with initial separation $d=4$ and inclinations $\Theta=0(a-c)$ and $\Theta=\pi / 2(d-f)$. Evolution of the centre-to-centre distance $(a, d)$, the radial component of the relative velocity $(b, e)$ and the deformation parameter $(c, f)$. Black dots correspond to the numerical simulations. Red line corresponds to the radial velocity and separation predicted by (4.7) and the blue line is the deformation of an isolated drop calculated from (5.2). The insets show the sign reversal of the radial velocity.

Figures 7 and 8 show that upon application of the field the drops deform into an oblate or prolate ellipsoid depending on the Taylor discriminating function. The deformation parameter increases monotonically, similarly to the isolated drop case, and approaches a nearly steady value, which is close to that for an isolated drop given by (5.1). Due to the axial symmetry, the deformation parameters of both drops are identical. The difference between the two drop and the isolated drop results is greater in the $\Theta=0$ case because as the drops are moving closer their interaction is getting stronger; in the $\Theta=\pi / 2$ configuration, the drops move away from each other and become more isolated. The strengthening interaction as separation decreases in the $\Theta=0$ case leads to an unsteady increase in the deformation parameter because the drop shapes lose fore-aft symmetry and deform greatly before contact.

The effect of the initial misalignment of the drop pair and the applied field direction is illustrated in figure 9 with the three-dimensional trajectory of drops in the two canonical cases $R / S<1$ and $R / S>1$. While in most cases drops display monotonic separation or attraction, figure 9 highlights some more intriguing dynamics: repulsion followed by attraction with centreline rotating towards the applied field direction $(a, d)$, attraction followed by repulsion with centreline rotating towards the applied field direction $(c)$ and attraction followed by repulsion with centreline rotating away from the applied field direction $(b)$. The drops remain in the plane defined by the initial separation vector and the applied field direction, in this case the $x z$ plane. The transient pair dynamics is clearly seen in the trajectories in the $x z$ plane.The unsteady drop interactions are illustrated in more detail in figure 10. In the systems $R=0.1$ and $S=1(\Phi>0), R=1$ and $S=10$ $(\Phi<0), R=1$ and $S=0.1(\Phi>0)$, the EHD interactions are dominant; in particular, for a sphere, $R=1$ completely switches off the DEP interaction. In the $R=1$ and $S=10$ $(\Phi<0)$ case (figure $10 c, d)$, the initial drop centreline angle is below $\Theta_{c}$ and the EHD interaction is attractive. The drops initially attract along the direction of the electric field, but the rotation of the centreline away from the field axis increases the tilt angle above 

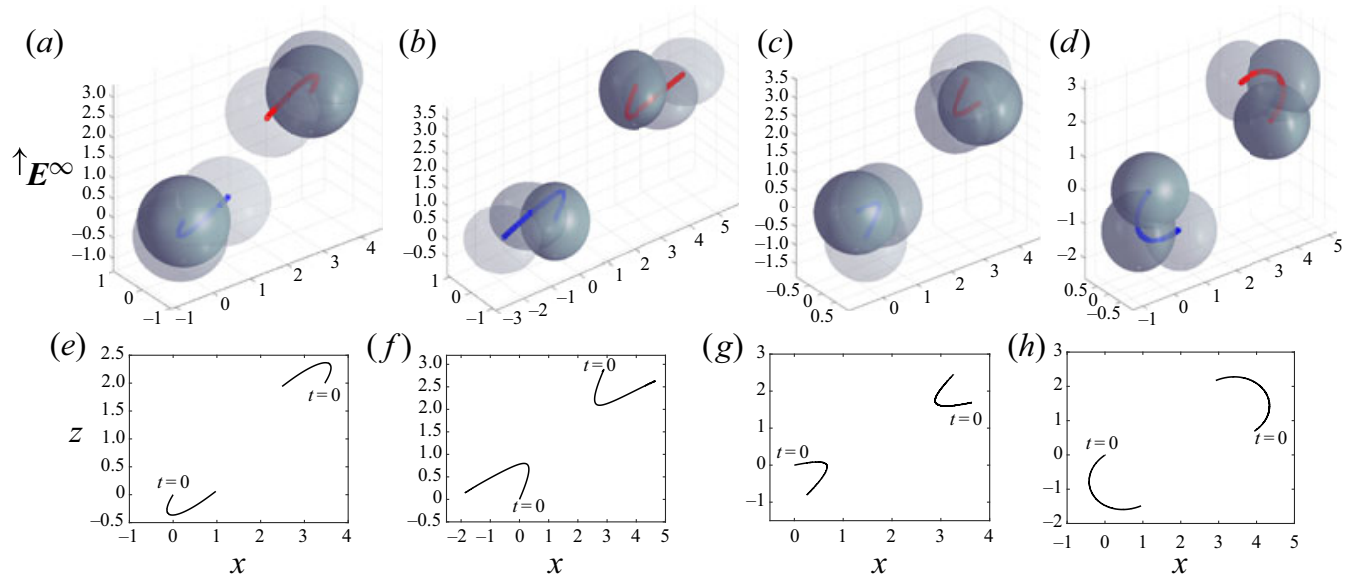

(g)
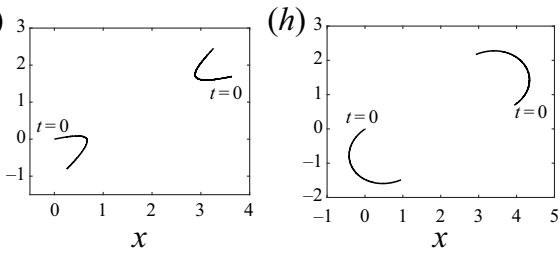

Figure 9. Trajectories of two identical drops with $(a, e) R=0.1, S=1,(b, f) R=1, S=10,(c, g) R=1$, $S=0.1$ and $(d, h) R=100, S=1$. Initially, the separation in all cases is $d=4$ and the angle with the applied field direction is $(a, e) \Theta=60^{\circ},(b, f) \Theta=45^{\circ},(c, g) \Theta=65^{\circ}$ and $(d, h) \Theta=80^{\circ}$. $(e, f, g, h)$ Trajectories in the $x z$ planes.

$\Theta_{c}$ leading to repulsion and separation in direction perpendicular to the field. The angle between the separation vector and the applied field continuously increases and around $65^{\circ}$ the interaction changes from attractive to repulsive. At this point the drops attain minimum separation, and after that the drops move away from each other with a velocity that overshoots. At long times the drop pair approaches a nearly perpendicular orientation relative to the field direction, where the repulsive DEP and EHD interactions push the drops apart. This 'kiss-and-run' dynamics is similar to the those observed with ideally polarizable spheres (Saintillan 2008) and has implications for electrocoalescence since the switching from attraction to repulsion prevents drops from reaching proximity sufficient to initiate merger. In all other cases, for which $\Phi>0$, drops move to align with the field. Figures $10(a, b)$ and $10(e, f)$ illustrate the repulsion/attraction and attraction/repulsion dynamics. In both cases, the drop is released at an initial angle above the critical, but the $R=0.1$ and $S=1$ EHD stresslet flow is repulsive, while the $R=1$ and $S=0.1$ EHD flow is attractive. Since $\Phi>0$, the drop centreline rotates towards the field direction bringing the drops into the range of angles where the EHD flow causes the drop interaction to reverse sign.

The DEP interactions become very important for large conductivity ratios $R \gg 1$. As $R$ increases the EHD flow weakens (see (4.3)), while the DEP force plateaus as the dipole strength $(R-1) /(R+2)$ approaches 1 (see (4.2)). As a result, the cross-over separation beyond which the EHD flow becomes important increases. The $R=100, S=1$ case (figure $10 g, h$ ) illustrates the dynamics in this DEP dominated regime. Choosing an initial angle larger than $\Theta_{c}$ causes the drops to repel, but $\Phi>0$ means that $\Theta$ will decrease with time below $\Theta_{c}$ and the drops will then attract.

\section{Conclusions and outlook}

The three-dimensional interactions of a drop pair in an applied electric field are studied using numerical simulations and a small-deformation theory based on the leaky dielectric model. We present results for the case of a uniform electric field and arbitrary 

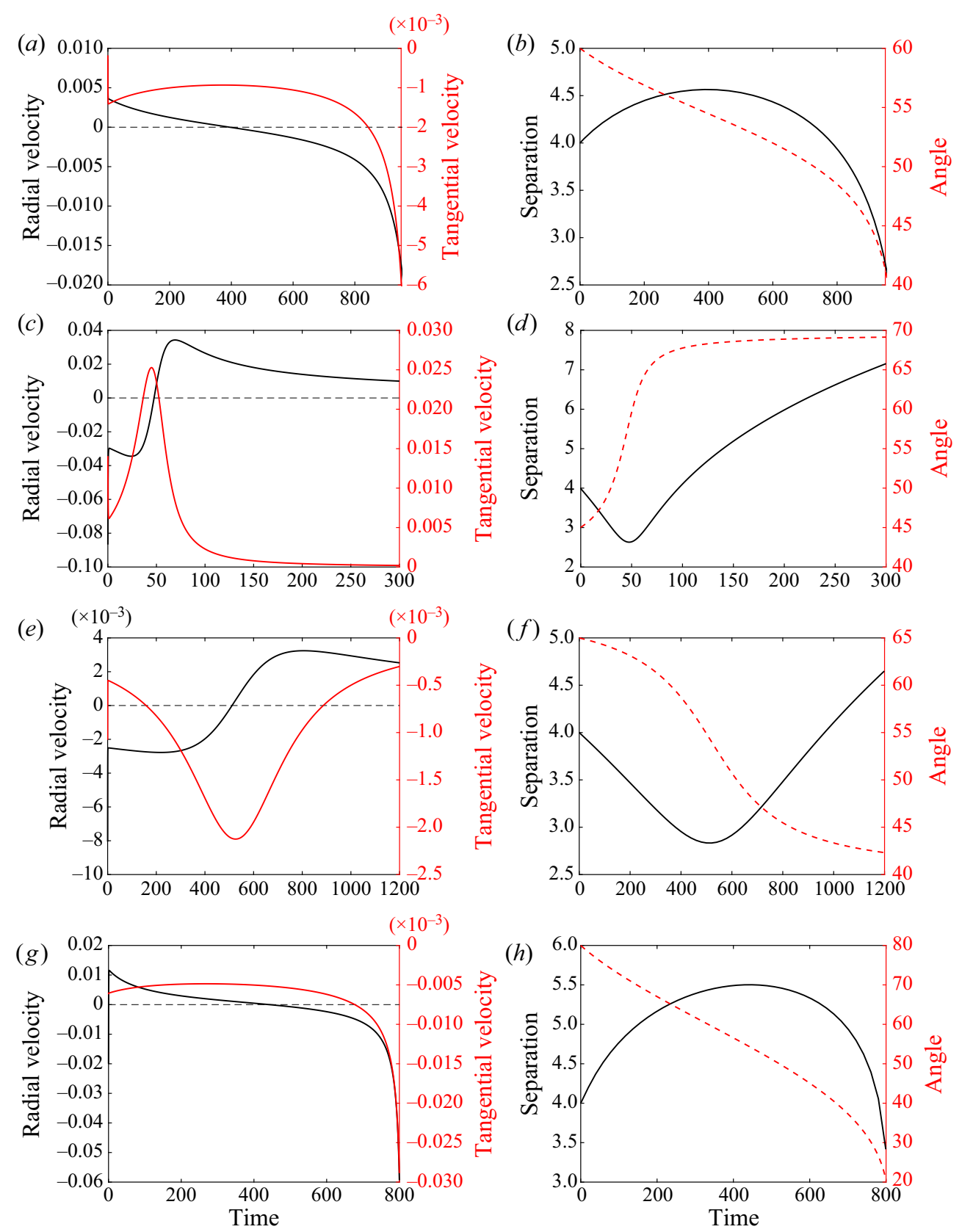

Figure 10. Dynamics of a pair of identical drops with initial separation $d=4$ and different angles with the applied field; $(a, b) R=0.1, S=1$ (repulsion-attraction, alignment with the field), $(c, d) R=1, S=10$ (attraction-repulsion, misalignment with the field), $(e, f) R=1, S=0.1$ (attraction-repulsion, alignment perpendicular to the field) and $(g, h) R=100, S=1$ (repulsion-attraction, alignment with the field).

angle between the drops' line of centres and the applied field direction, where the non-axisymmetric geometry necessitates three-dimensional simulations.

The pair dynamics depends on the interplay between the EHD and DEP interactions, which are cooperative in the case of $R / S<1$, and antagonistic for $R / S>1$. 


\title{
C. Sorgentone and others
}

DEP interaction favours drop pair alignment with the field and is attractive for small angles and repulsive otherwise. The critical angle where centre-to-centre motion changes sign can be estimated from the point-dipole approximation, $\Theta_{c}=\arccos (1 / \sqrt{3}) \approx 54.7^{\circ}$. The EHD interaction depends on the sign of the induced free-charge dipole, which is dictated by the difference $\sim(R-S)$. If $R / S<1$, the pole to equator flow pulls the drops together when aligned parallel to the applied field direction and pushes them apart when the centre-to-centre line is perpendicular to the field; this scenario reverses for $R / S>1$. The critical angle which separates attraction from repulsion can be estimated from the stresslet approximation of the EHD flow and is the same as the DEP force. Hence, to leading order in separation and drop deformation, both the DEP and EHD change sign at $\Theta_{c}$. Unlike DEP, the EHD interaction can cause the drops' line of centres to rotate toward or away from the applied field direction. The theory highlights the importance of the function $\Phi(\lambda, R, S)$, given by (4.8), which discriminates between the drop pair moving to align with the field or in a direction transverse to the field.

Our study finds that if the drop pair angle with the field initially is close to the critical angle for reversal of the interaction sign, the drops do not experience monotonic attraction or repulsion; instead their trajectories follow three scenarios: motion in the direction of the field accompanied by either attraction followed by separation or vice versa (repulsion followed by attraction), and attraction followed by separation in a direction transverse to the field. The dynamics of drops with $R / S<1$ and $\Phi<0$ is similar to ideally polarizable spheres (Saintillan 2008) due to the similarities of the flow pattern (despite different flow origins): the drops attract and move in the direction of the field and then separate in the transverse direction. Hence, coalescence will be prevented in such cases. Drops with $R / S>1$ tend to align with the field but the sign of the interaction depends on drop separation. The DEP dominates when drops are close, while EHD controls the far-field interaction.

The comparison of the analytical theory and the simulations shows that the theory performs quite well in a wide range of drop separations and angles with the applied field direction for $C a<1$, and thus can serve as an efficient means to estimate drop pair dynamics and trajectories in an applied electric field. However, the simulations are indispensable in modelling the near-contact motions of the drops and the drop dynamics in stronger fields. Our three-dimensional boundary integral method is also capable of simulating the dynamics of dissimilar drops (different size, viscosities, $R$ and $S$ ), and many drops, which we plan to explore in the future. Charge convection can also be included in order to study symmetry-breaking three-dimensional instabilities such as the Quincke electrorotation (Salipante \& Vlahovska 2010, 2013; Vlahovska 2016b).

Supplementary movies. Supplementary movies are available at https://doi.org/10.1017/jfm.2020.1007.

Acknowledgements. We thank the anonymous referees for critical reading and suggestions.

Funding. C.S. gratefully acknowledges support by Comsol Inc. P.V. has been supported in part by NSF award CBET-1704996. J.K., A.K. and L.W. have been supported by NSF award CBET-1804548.

Declaration of interests. The authors report no conflict of interest.

\author{
Author ORCIDs. \\ (1) Aditya S. Khair https://orcid.org/0000-0001-6076-2910; \\ Detia M. Vlahovska https://orcid.org/0000-0001-7549-930X.
}

914 A24-16 


\section{Electrohydrodynamic interaction of drop pairs}

\section{Appendix A. Evolution of the drops velocity upon application of an uniform electric field}

Let us consider the transient drop dynamics after the electric field is applied in the limit of small deformations $C a \ll 1$. At leading order in $C a$, the shape is described by $r_{s}=$ $1+f_{2}(t)\left(-1+3 \cos ^{2} \theta\right)$, and the velocity field outside the drop at distance $r$ from the drop centre and an angle $\theta$ with the applied field direction is given by Vlahovska (2011, $2016 a$ )

$$
\boldsymbol{u}=\left(\frac{\alpha+\beta}{r^{2}}-\frac{\beta}{r^{4}}\right)\left(-1+3 \cos ^{2} \theta\right) \hat{\boldsymbol{r}}-\frac{\beta}{r^{4}} \sin (2 \theta) \hat{\boldsymbol{\theta}} .
$$

The coefficients $\alpha$ and $\beta$ are time dependent because the drop deforms

$$
\left.\begin{array}{c}
\alpha(t)=\frac{15(\lambda+1)}{(3+2 \lambda)(16+19 \lambda)}\left(F_{T}(R, S, \lambda)-C a^{-1} \frac{8}{3} f_{2}(t)\right), \\
(t)=\frac{1}{(3+2 \lambda)(16+19 \lambda)}\left(B_{T}(R, S, \lambda)-C a^{-1} f_{2}(t)(12(2+3 \lambda))\right),
\end{array}\right\}
$$

where $F_{T}$ is the Taylor discriminating function

$$
F_{T}(R, S, \lambda)=\frac{1}{(2+R)^{2}}\left(R^{2}+1-2 S+3(R-S) \frac{2+3 \lambda}{5(\lambda+1)}\right),
$$

and

$$
B_{T}(R, S, \lambda)=\frac{9\left(\lambda\left(3 R^{2}+13 R-19 S+3\right)+2\left(R^{2}+6 R-8 S+1\right)\right)}{2(R+2)^{2}} .
$$

The shape evolution equation is obtained from the kinematic condition $\dot{r}_{s}=u_{r}(r=1)$

$$
\dot{f}_{2}=\frac{15(\lambda+1)}{(3+2 \lambda)(16+19 \lambda)}\left(F_{T}(R, S, \lambda)-C a^{-1} \frac{8}{3} f_{2}(t)\right) .
$$

Note that the Taylor deformation parameter is related to $f_{2}, D=(3 / 2) f_{2}$, which leads to (5.2) describing the transient shape of an isolated drop.

If a second drop is present at location $x_{2}^{c}=d \hat{d}$, its migration velocity due to the EHD flow of the first drop can be obtained using Faxen's law (Kim \& Karrila 1991)

$$
\boldsymbol{U}_{2}^{e h d}=\left(1+\frac{\lambda}{2(3 \lambda+2)} \nabla^{2}\right) \boldsymbol{u}(r=d) .
$$

Inserting (A1) in the above equation yields

$$
\left.\begin{array}{c}
U_{2, r}^{e h d}=\left(\frac{\alpha+\beta}{d^{2}}-\frac{1}{d^{4}}\left(\beta+\frac{3 \lambda}{2+3 \lambda}(\alpha+\beta)\right)\right)\left(-1+3 \cos ^{2} \Theta\right), \\
U_{2, t}^{e h d}=-\frac{1}{d^{4}}\left(\beta+\frac{3 \lambda}{2+3 \lambda}(\alpha+\beta)\right) \sin (2 \Theta),
\end{array}\right\}
$$

where $\Theta=\arccos (\hat{\boldsymbol{d}} \cdot \hat{\boldsymbol{z}})$. At steady state $f_{2}=(3 / 8) C a F_{T}, \alpha=0$ and $\beta$ reduces to Taylor's result

$$
\beta_{T}=\frac{9(R-S)}{10(1+\lambda)(2+R)^{2}},
$$

which leads to the steady EHD contribution to the migration velocity calculated assuming a spherical drop (4.6). Recall that, for a perturbation solution in $\mathrm{Ca}$, the leading-order 


\section{Sorgentone and others}
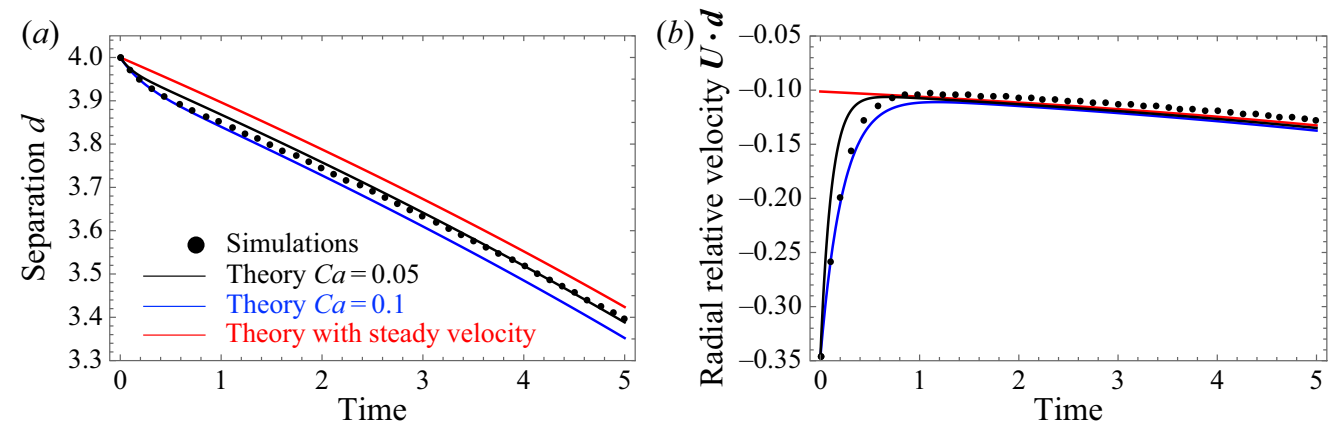

Figure 11. Comparison between the transient and steady migration theories with the numerical simulations. Centroid distance $(a)$ and relative radial velocity $(b)$ as a function of time for a pair of identical drops with $R=1, S=10$, aligned with the field $(\Theta=0)$ and initial separation $d=4, C a=0.1$. The red line corresponds to the trajectory computed using the steady state velocity (4.7). The transient solution using (A9) with $\mathrm{Ca}=$ 0.05 is given in black and $\mathrm{Ca}=0.1$ in blue.

steady flow about the deformed drop is identical to the solution for a spherical drop (Rallison 1980).

The relative drop velocity is obtained by adding the contribution from the DEP force, (4.2), which is time-independent

$$
\boldsymbol{U}(t)=2\left(\boldsymbol{U}_{2}^{e h d}(t)+\boldsymbol{U}_{2}^{\text {dep }}\right) .
$$

Figure 11 compares trajectories computed from a velocity that is transient (in the case of a deforming drop) and steady (in the case of a spherical drop). Decreasing $C a$ shortens the transient period and the trajectory approaches the steady result. However, a long transient results in an offset.

\section{REFERENCES}

AnAND, V., Roy, S., NAik, V.M., JuvekaR, V.A. \& ThaOkaR, R.M. 2019 Electrocoalescence of a pair of conducting drops in an insulating oil. J. Fluid Mech. 859, 839-850.

Baygents, J.C., Rivette, N.J. \& Stone, H.A. 1998 Electrohydrodynamic deformation and interaction of drop pairs. J. Fluid Mech. 368, 359-375.

Brosseau, Q. \& Vlahovska, P.M. 2017 Streaming from the equator of a drop in an external electric field. Phys. Rev. Lett. 119, 034501.

Casas, P.S., Garzon, M., Gray, L.J. \& Sethian, J.A. 2019 Numerical study on electrohydrodynamic multiple droplet interactions. Phys. Rev. E 100, 063111.

COllins, R.T., JONES, J.J., HARRIS, M.T. \& BASARAN, O.A. 2008 Electrohydrodynamic tip streaming and emission of charged drops from liquid cones. Nat. Phys. 4, 149-154.

Collins, R.T., Sambath, K., Harris, M.T. \& Basaran, O.A. 2013 Universal scaling laws for the disintegration of electrified drops. Proc. Natl Acad. Sci. 110, 4905-4910.

DAS, D. \& SAINTILlan, D. 2017 Electrohydrodynamics of viscous drops in strong electric fields: numerical simulations. J. Fluid Mech. 829, 127-152.

DonG, Q. \& SAU, A. 2018 Electrohydrodynamic interaction, deformation, and coalescence of suspended drop pairs at varied angle of incidence. Phys. Rev. Fluids 3, 073701.

EOW, J.S. \& GHADIRI, M. 2002 Electrostatic enhancement of coalescence of water droplets in oil: a review of the technology. Chem. Engng Sci. 85, 357-368.

Esmaeeli, A. \& ShARIFI, P. 2011 Transient electrohydrodynamics of a liquid drop. Phys. Rev. E 84, 036308.

FERNANDEZ, A. $2008 a$ Response of an emulsion of leaky dielectric drops immersed in a simple shear flow: drops less conductive than the suspending fluid. Phys. Fluids 20, 043304.

FERNANDEZ, A. $2008 b$ Response of an emulsion of leaky dielectric drops immersed in a simple shear flow: drops more conductive than the suspending fluid. Phys. Fluids 20, 043303. 


\section{Electrohydrodynamic interaction of drop pairs}

Ganan-Calvo, A.M., Lopez-Herrera, J.M., Herrada, M.A., Ramos, A. \& Montanero, J.M. 2018 Review on the physics of electrospray: from electrokinetics to the operating conditions of single and coaxial Taylor cone-jets, and ac electrospray. J. Aerosol Sci. 125, 32-56.

Ganan-Calvo, A.M., Lopez-Herrera, J.M., Rebollo-Munoz, N. \& Montanero, J.M. 2016 The onset of electrospray: the universal scaling laws of the first ejection. Sci. Rep. 6, 32357.

HA, J.W. \& YANG, S.M. 2000 Electrohydrodynamics and electrorotation of a drop with fluid less conductive than that of the ambient fluid. Phys. Fluids 12, 764-772.

Herrada, M.A., López-Herrera, J.M., Gañán Calvo, A.M., Vega, E.J., Montanero, J.M. \& Popinet, S. 2012 Numerical simulation of electrospray in the cone-jet mode. Phys. Rev. E 86, 026305 .

KARYAPPA, R.B., DEShMukh, S.D. \& ThAOKAR, R.M. 2014 Breakup of a conducting drop in a uniform electric field. J. Fluid Mech. 754, 550-589.

Kennedy, C.A. \& CARPEnTER, M.H. 2003 Additive Runge-Kutta schemes for convection-diffusionreaction equations. Appl. Numer. Maths 44 (1), 139-181.

Kim, S. \& Karrila, S.J. 1991 Microhydrodynamics: Principles and Selected Applications. Butterworth-Heinemann.

LAC, E. \& HoMSY, G.M. 2007 Axisymmetric deformation and stability of a viscous drop in a steady electric field. J. Fluid Mech. 590, 239-264.

LANAUZE, J.A., WALKer, L.M. \& KHAIR, A.S. 2015 Nonlinear electrohydrodynamics of slightly deformed oblate drops. J. Fluid Mech. 774, 245-266.

Lin, Y., SkJETne, P. \& CARLson, A. 2012 A phase field model for multiphase electro-hydrodynamic flow. Intl J. Multiphase Flow 45, 1-11.

MELChER, J.R. \& TAYLOR, G.I. 1969 Electrohydrodynamics - a review of role of interfacial shear stress. Annu. Rev. Fluid Mech. 1, 111-146.

Mhatre, S., Deshmukh, S. \& Thaokar, R.M. 2015 Electrocoalescence of a drop pair. Phys. Fluids 27 (9), 092106.

DE LA MorA, J.F. 2007 The fluid dynamics of Taylor cones. Annu. Rev. Fluid Mech. 39, 217-243.

MoRI, Y. \& YounG, Y.-N. 2018 Electrohydrodynamics of leaky dielectrics as the weak electrolyte limit of an electrodiffusion model. J. Fluid Mech. 855, 67-130.

Pillai, R., BERRY, J.D., HARVIE, D.J.E. \& DAVIDSON, M.R. 2016 Electrokinetics of isolated electrified drops. Soft Matt. 12, 3310-3325.

Rahimian, A., VeErapaneni, S.K., Zorin, D. \& Biros, G. 2015 Boundary integral method for the flow of vescicles with viscosity contrast in three dimensions. J. Comput. Phys. 298, 766-786.

RAllison, J.M. 1980 Note on the time-dependent deformation of a viscous drop which is almost spherical. J. Fluid Mech. 98, 625-633.

RoY, S., ANAND, V. \& THAOKAR, R.M. 2019 Breakup and non-coalescence mechanism of aqueous droplets suspended in castor oil under electric field. J. Fluid Mech. 878, 820-833.

Saintillan, D. 2008 Nonlinear interactions in electrophoresis of ideally polarizable particles. Phys. Fluids 20 (6), 067104.

Salipante, P.F. \& Vlahovska, P.M. 2010 Electrohydrodynamics of drops in strong uniform dc electric fields. Phys. Fluids 22, 112110.

Salipante, P.F. \& Vlahovska, P.M. 2013 Electrohydrodynamic rotations of a viscous droplet. Phys. Rev. E 88, 043003.

SAVILlE, D.A. 1997 Electrohydrodynamics: the Taylor-Melcher leaky dielectric model. Annu. Rev. Fluid Mech. 29, 27-64.

SCHNITZER, O. \& YARIV, E. 2015 The Taylor-Melcher leaky dielectric model as a macroscale electrokinetic description. J. Fluid Mech. 773, 1-33.

Sengupta, R., WAlker, L.M. \& Khair, A.S. 2017 The role of surface charge convection in the electrohydrodynamics and breakup of prolate drops. J. Fluid Mech. 833, 29-53.

SHERwOOD, J.D. 1988 Breakup of fluid droplets in electric and magnetic fields. J. Fluid Mech. 188, $133-146$.

Sorgentone, C. \& TORnBERG, A.-K. 2018 A highly accurate boundary integral equation method for surfactant-laden drops in 3D. J. Comput. Phys. 360, 167-191.

Sorgentone, C., Tornberg, A.-K. \& Vlahovska, P.M. 2019 A 3D boundary integral method for the electrohydrodynamics of surfactant-covered drops. J. Comput. Phys. 389, 111-127.

Sozou, C. 1975 Electrohydrodynamics of a pair of liquid drops. J. Fluid Mech. 67 (2), 339-348.

Stone, H.A., Stroock, A.D. \& AJdARI, A. 2005 Engineering flows in small devices: microfluidics toward a lab-on-a-chip. Annu. Rev. Fluid Mech. 36, 381-411.

TAYlor, G.I. 1964 Disintegration of water drops in an electric field. Proc. R. Soc. Lond. A 280 (1382), 383-397. 


\section{Sorgentone and others}

TAYLOR, G.I. 1966 Studies in electrohydrodynamics. I. Circulation produced in a drop by an electric field. Proc. R. Soc. Lond. A 291, 159-166.

TorzA, S., Cox, R.G. \& MASON, S.G. 1971 Electrohydrodynamic deformation and burst of liquid drops. Phil. Trans. R. Soc. Lond. A 269, 295-319.

VeErapaneni, S.K., Rahimian, A., Biros, G. \& Zorin, D. 2011 A fast algorithm for simulating vesicle flows in three dimensions. J. Comput. Phys. 230, 5610-5634.

VlahovsKa, P.M. 2011 On the rheology of a dilute emulsion in a uniform electric field. J. Fluid Mech. 670, 481-503.

VLAhOVSKA, P.M. $2016 a$ Dynamics of membrane bound particles: capsules and vesicles. In Low-Reynolds-Number Flows: Fluid-Structure Interactions (ed. C. Duprat \& H.A. Stone). Royal Society of Chemistry Series RSC Soft Matter.

Vlahovska, P.M. $2016 b$ Electrohydrodynamic instabilities of viscous drops. Phys. Rev. Fluids 1, 060504.

Vlahovska, P.M. 2019 Electrohydrodynamics of drops and vesicles. Annu. Rev. Fluid Mech. 51, 305-330.

Vlahovska, P.M., Gracia, R.S., Aranda-EspinOza, S. \& Dimova, R. 2009 Electrohydrodynamic model of vesicle deformation in alternating electric fields. Biophys. J. 96, 4789-4803.

WAGONer, B.W., Vlahovska, P.M., Harris, M.T. \& BASARAn, O.A. 2020 Electric field induced transitions from spherical to discocyte and lens shaped drops. J. Fluid Mech. 904, R4.

WANG, Q., MA, M. \& Siegel, M. 2019 Deformation and stability of a viscous electrolyte drop in a uniform electric field. Phys. Rev. Fluids 4, 053702.

YING, L., BIROS, G. \& ZoRIN, D. 2006 A high-order 3D boundary integral equation solver for elliptic PDEs in smooth domains. J. Comput. Phys. 219 (1), 247-275.

ZABARANKIn, M. 2020 Small deformation theory for two leaky dielectric drops in a uniform electric field. Proc. R. Soc. Lond. A 476 (2233), 20190517. 